\section{P11 PLASMA MEMBRANE CALCIUM ATPASE 1 GENE EXPRESSION INCREASES IN VASCULAR SMOOTH MUSCLE CELLS TREATED WITH INDUCERS OF PULMONARY ARTERIAL HYPERTENSION}

\begin{abstract}
'Jude C Ihugba, ' $S a t h i s h k u m a r$ Kurusamy, ${ }^{2}$ Nadine Arnold, ${ }^{1}$ Priscille Polla, ${ }^{3,4,5}$ Pablo Gomez-del Arco, ${ }^{6}$ Emanuel E Strehler, ${ }^{4,5}$ Juan Miguel Redondo, ${ }^{7} J a m e s$ Cotton, ${ }^{2}$ Allan Lawrie, ${ }^{1}$ Angel Luis Armesilla. ${ }^{1}$ Cardiovascular Molecular Pharmacology Laboratory, School of Pharmacy, Research Institute in Healthcare Science, Faculty of Science and Engineering, University of Wolverhampton, Wolverhampton, UK; ${ }^{2}$ Pulmonary Vascular Research Group, Infection, Immunity and Cardiovascular Disease, University of Sheffield, Sheffield, UK; ${ }^{3}$ Gene Regulation in Cardiovascular Remodelling and Inflammation Group, Centro Nacional de Investigaciones Cardiovasculares, Madrid, Spain; ${ }^{4}$ CIBERCV; ${ }^{5}$ Department of Molecular Biology, Universidad Autonoma de Madrid, CBM-SO, Madrid, Spain; ${ }^{6}$ Department of Biochemistry and Molecular Biology, Mayo Clinic College of Medicine, Rochester, USA; ${ }^{7}$ Department of Cardiology, Heart and Lung Centre, New Cross Hospital, Wolverhampton, UK
\end{abstract}

\subsection{6/heartjnl-2018-BSCR.16}

Pulmonary arterial hypertension (PAH) is a chronic and lifethreatening disease characterised by a progressive narrowing and occlusion of small pulmonary arteries leading to increased pulmonary resistance, right ventricular hypertrophy, and, finally, right ventricular failure.

Several studies have demonstrated that proliferation and migration of pulmonary arterial smooth muscle cells (PASMCs) play a pivotal role in the vascular remodelling characteristic of PAH. Levels of cytoplasmic calcium are an important determinant of PASMC proliferation and migration. The Plasma Membrane Calcium ATPase (PMCA) proteins extrude calcium from the cytosol to the extracellular medium.

Here, we have investigated whether inducers of PAH trigger any changes in the expression of PMCA genes in PASMCs. Treatment of PASMCs with the PAH inducers Platelet Derived Growth Factor (PDGF) or TNF-alpha induced a significant increase in the RNA levels of PMCA1. PMCA1 RNA levels are also elevated in lungs of rats with monocrotaline-induced PAH. In silico analysis of the PMCA1 gene promoter region has shown putative binding sites for the transcription factors NFAT and NFkB that could mediate the transcriptional upregulation triggered by PDGF and TNF-alpha respectively. However, we show here that upregulated PMCA1 gene expression is not mediated by binding of these transcription factors to the proximal promoter region. No changes were observed in the RNA levels of PMCA4, the other major PMCA isoform expressed in PASMCs.

Our results suggest an important role for PMCA1 in PASMC deregulation during PAH, although a full understanding of the role of PMCA1 on the onset and progression of $\mathrm{PAH}$ requires further investigation.

\section{P12 A NOVEL MODEL OF CARDIOMYOPATHY REVEALS A TISSUE SPECIFIC ROLE FOR THE COMPLEX I ASSEMBLY FACTOR ECSIT}

${ }^{1} \mathrm{~T}$ Nicol, ${ }^{1} \mathrm{~S}$ Falcone, ${ }^{1} \mathrm{~A}$ Blease, ${ }^{1} \mathrm{C}$ Scudamore, ${ }^{2} \mathrm{f}$ Hirst, ${ }^{2} \mathrm{C}$ Viscomi, ${ }^{2} \mathrm{M}$ Zeviani, ${ }^{1}$ SDM Brown, ${ }^{1}$ PK Potter. ${ }^{1}$ Medical Research Council Harwell Institute, Harwell, Oxfordshire; ${ }^{2}$ Medical Research Council Mitochondrial Biology Unit, Cambridge

10.1136/heartjnl-2018-BSCR.17

Here we present a mouse model with a missense mutation in the gene Ecsit that shows a progressive cardiomyopathy from 4 weeks of age with no other overt phenotypes. ECSIT is known to play a role in development and immune signalling but is also thought to function as an assembly factor of complex I.

Western blot analysis of tissue lysates revealed a significant reduction in complex I proteins in heart tissue, whereas all other complexes were unaffected. In addition, Seahorse analysis of isolated mitochondria shows a significant reduction in the respiration rates of cardiac mitochondria, whilst no differences could be seen in mitochondria isolated from brain tissue.

In-gel activity demonstrated a significant drop in complex I activity of cardiac mitochondria, whilst brain mitochondria are maintained at close to normal levels. Blue native PAGE performed on cardiac mitochondria shows that this mutation affects ECSIT's role in a limited number of complex I subassemblies. However, this is unique to the heart and mitochondria from brain tissue show no changes in any of the same sub-assemblies, supporting the initial findings that there is normal complex I assembly in the brain.

A potential mechanism lies in the discovery of a previously undescribed $16 \mathrm{kDa}$ fragment of ECSIT that is present in WT cardiac mitochondria but not in mutant. This fragment is also undetectable in mitochondria isolated from brain tissue, indicating a tissue specific cleavage of ECSIT protein as a method of action.

\section{P13 CHANGES IN MITOCHONDRIAL MORPHOLOGY \& DISTRIBUTION DURING POSTNATAL DEVELOPMENT}

K Summers, H Lin, M Caputo, MS Suleiman. School of Clinical Sciences, University of Bristol, Tyndall Avenue, Bristol

10.1136/heartjnl-2018-BSCR. 18

Vulnerability to cardiac ischemia/reperfusion injury (I/RI) changes during postnatal development, with greatest resistance at postnatal day 14 (P14) in rats. The underlying mechanism is currently unknown. We hypothesise that developmental changes in mitochondrial morphology and distribution of the 3 mitochondrial subpopulations - interfibrillar, perinuclear and subsarcolemmal- may be linked to these differences in vulnerability.

Hearts from P14 $(n=3)$ and adult $(n=3)$ rats were perfused with Krebs-solution, followed by fixative solution (1\% glutaraldehyde, $1 \%$ paraformaldehyde), before processing and embedding in EPON epoxy resin for electron microscopy. Images were taken using a Tecnai12 TEM electron microscope, from which areas, widths, lengths and densities of mitochondria from the 3 subpopulations were measured using Image J.

All 3 mitochondrial subpopulations had statistically significantly larger mean area, lower aspect ratio, and greater roundness in adult compared with P14 hearts. Adult hearts had a significantly higher density of interfibrillar but lower density of perinuclear mitochondria than P14 hearts. There were also differences in subpopulations within each age group. At P14, interfibrillar mitochondria had a greater area but lower roundness in comparison with perinuclear and subsarcolemmal mitochondria, the two of which showed no significant difference from one another. In adults however, only interfibrillar and subsarcolemmal mitochondria showed significant differences in area, whereas all 3 subpopulations differed in roundness.

These data demonstrate marked differences in mitochondrial morphology and distribution between P14 and adult 